\title{
LA EVALUACIÓN DEL ESTRÉS Y DEL TRAUMA: PRESENTACIÓN DE LA VERSIÓN ESPAÑOLA DE LA ESCALA DE TRAUMA ACUMULATIVO (CTS)
}

Robles, M. E., Badosa, J. M., Roig

VIAbcn, Barcelona

A. Pina, B.

Feixas, G.

Universitat de Barcelona

This paper reviews some of the most usual definitions of stress and trauma including the notion of accumulative trauma. Then, the Spanish version of the Cumulative Trauma Scale by Kira et al (2008) is presented. It has been developed by following a process of translation and by applying it to two small groups of subjects to test it for comprehension (pilot test).

Keywords: Trauma, stress

Se ha puesto mucho énfasis en la literatura científica del estrés y el trauma en explicar y entender los mediadores y moderadores que influyen en la respuesta de nuestro cuerpo ante las situaciones que nos resultan amenazadoras o desafiantes con el fin de conseguir mejores métodos de evaluación, intervención y prevención (Dulmus \& Hilarski, 2003). Aún así, el lector suele confundirse en el uso de la terminología, ya que normalmente se intercambian los conceptos (Lewis \& Roberts, 2002).

Estrés

El estudio del estrés incluye un gran número de diferentes perspectivas y descripciones del mismo. Desde entenderlo como una respuesta a la situación

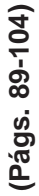
ambiental (Selye, 1950, 1973), un desafío del contexto situacional (Dohrenwend, 2000), la relación entre las demandas del medio ambiente y la capacidad de satisfacer dichas demandas (Taylor \& Roberts, 1995), hasta una relación particular 
entre una persona y el contexto que es apreciada por la persona como superior a sus recursos (Lazarus, 1984). Más recientemente, se ha planteado que el ser humano cuenta con un sistema de procesamiento de valores e interiorización previos a la presentación de un acontecimiento. Este sistema se activa de forma automática ante el acontecimiento y pone en marcha la activación o inhibición del procesamiento emocional y cognitivo, así como un estado de alerta y de respuesta (Kira, 2001). Con diferentes matices, estas definiciones entienden el estrés como una percepción (Resick, 2001); así, el mismo acontecimiento activará diferentes respuestas en diferentes personas.

\section{Trauma}

Algo similar sucede en el caso del trauma. Usualmente esta palabra se refiere tanto a los eventos negativos que producen malestar como al propio malestar en sí (Briere \& Scott, 2006). Técnicamente, trauma se refiere sólo al evento, no a la reacción. Para el DSM-IV-TR (APA, 2002) un evento traumático se limita a hechos que representan un peligro real para la vida o cualquier otra amenaza para la integridad física (p. 518). Esta limitación ha generado cierta controversia (Bracken, 2002; Gorman, 2001; Lykes, 2000; North, et al., 2009; Ray, 2008; Summerfield, 2004), puesto que muchos eventos pueden ser traumáticos aunque no exista amenaza vital o daño físico (Briere \& Spinazzola, 2005). Según esta definición, quedarían excluidos de ser considerados como trauma la humillación, las pérdidas significativas o el abuso emocional extremo (Ford et al., 2006; Giacaman et al., 2007).

Existe un mayor consenso en entender el trauma psicológico como una experiencia súbita e inesperada, que excede la capacidad individual percibida del sujeto para satisfacer lo demandado y tiene como resultado la alteración del marco de referencia propio (Linden, 2007; McCann \& Pearlman, 1990). Es decir, un incidente puede ser considerado traumático cuando los recursos internos del sujeto no son capaces de asumir el impacto producido. Considerarlo así permite un mayor rango de acción a nivel terapéutico, al poder usar terapias focalizadas en el trauma, como la terapia cognitivo-conductual focalizada en el trauma o el EMDR (Ehlers et al., 2010), pero no modifica el ámbito diagnóstico, que sigue limitado a la propuesta del DSM. De esta manera, la teoría del trauma será un caso especial de la teoría de la respuesta de estrés; los sucesos traumáticos son la última o más grave clase de estrés (Kira, 2001).

\section{Clasificación estrés/trauma}

Existen tantas clasificaciones del continuo estrés/trauma como definiciones sobre estos conceptos. Dos de las más usadas son las que diferencian según el tipo de vivencia sufrida - directa o indirecta- y las que clasifican el estrés según su funcionalidad -adaptativa o desadaptativa-. 
En la primera clasificación, surge la diferenciación entre estrés primario directo- o estrés secundario -indirecto-. Así, el estrés primario es la vivencia de un incidente crítico de manera directa, entendiendo al propio sujeto como víctima. En contraste con el estrés primario, el estrés secundario se define como los comportamientos y las emociones naturales derivados de conocer acerca de un incidente traumático sufrido por un otro significativo o el estrés resultante de ayudar o desear ayudar a una persona que sufre o está traumatizada (Figley, 1995). Este tipo de estrés, también es conocido con el nombre de trauma vicario (McCann \& Pearlman, 1990) o fatiga por compasión (Figley, 1995). Otro constructo relacionado que se halla en la literatura es el burnout, que se caracteriza por un agotamiento físico, emocional y mental, además de despersonalización, causado por vivencias emocionalmente demandantes durante un tiempo prolongado (Pines \& Aronson, 1989). Estrés secundario y burnout se diferencian como constructos y parece que tienen distintos efectos sobre el bienestar profesional (Figley, 2002; Jenkins \& Baird, 2002; Robinson et al., 2003; Sabin-Farrell \& Turpin, 2003). Si se les atribuyera dominios afectivos a estos constructos, el estrés secundario podría establecerse cercano al miedo, a la ansiedad y a la depresión, y el burnout al cansancio emocional y a la sensación de falta de auto-eficacia (Figley, 2002; Larsen, Stamm, \& Davis, 2002).

En la segunda clasificación, el estrés se clasificaría en cuatro tipos: estrés general, estrés del incidente crítico, estrés postraumático y estrés acumulativo (Mitchell, 2003). Los dos primeros son presentaciones adaptativas del estrés. Así, el estrés general aparece frente a las demandas diarias de la vida y el estrés relacionado con el incidente crítico, también llamado estrés agudo, es una reacción estresante normal de una persona normal frente a una situación anormal. Se trata de una reacción displacentera, un estado de activación que se deriva de una exposición a un acontecimiento traumático de gran alcance (Flannery, 1999). En cambio, los otros dos tipos de estrés, tanto el postraumático como el acumulativo, son capaces de producir una ruptura en la vida de aquellos que sufren estas condiciones. Las características del postraumático parten de una triple sintomatología (Everly \& Mitchell, 1999). La re-experimentación incluye síntomas como flashbacks, recuerdos intrusivos, pesadillas y angustia cuando la persona se expone a estímulos relacionados con el incidente. La evitación incluye pensamientos o emociones fuera de sí mismo, así como la evitación de personas, lugares o incluso la incapacidad de hablar de ciertos aspectos del incidente. Y la hiperactivación se caracteriza por constantes reacciones de alerta, irritabilidad y alteraciones en el sueño (Briere, 1998).

En el estrés acumulativo se da una acumulación excesiva de estrés general sin resolver (Mitchell, 2003) y la exposición repetida frente al evento, aumenta la probabilidad de una respuesta patológica (van der Kolk, McFarlane yWeisaeth, 1996). En efecto, es la suma del número total de los factores de estrés, además de 
la gravedad de cada factor, más la longitud de tiempo en que el estrés se ha producido. Aquí se incluyen diferentes dinámicas que no están presentes en los modelos restantes, como son las dinámicas de acumulación y estimulación (kindling). El estrés acumulativo implica, al menos, dos tipos de experiencias estresantes, las nucleares y las desencadenadas por un acontecimiento. Las primeras sensibilizan y predisponen a un sesgo de respuestas de re-traumatización; y las desencadenadas por un acontecimiento son las que realmente ponen en marcha la respuesta postacumulativa, son la gota que colma el vaso (Kira et al., 2008).

Estas clasificaciones son complementarias entre sí. En efecto, por ejemplo, en un caso de estrés postraumático, dependiendo del tipo de exposición al trauma, directa como víctima o indirecta como cuidador, se clasificaría como estrés postraumático primario o secundario (Figley, 1995).

Más recientemente, Kira et al. (2008) ha propuesto dos clasificaciones diferentes o taxonomías de los estímulos psicológicos traumáticos. Cada una de estas taxonomías describe una dimensión diferente del incidente traumático. La primera, cubre las áreas de funcionamiento individual y clasifica las experiencias traumáticas según la función del desarrollo que queda afectada por el trauma. Así, se obtienen cinco tipos de áreas afectadas por un trauma: apego, identidad (personal o colectiva), interdependencia, autorrealización y supervivencia física. La segunda está basada en las características de los eventos traumáticos e incluyen el trauma acumulativo, el trauma interno (p.ej. dolor a consecuencia de un accidente automovilístico), el trauma provocado por la naturaleza (p.ej. huracanes) y el trauma provocado por el hombre. Este último se divide en traumas provocados por la persona y traumas provocados por la sociedad (p.ej. pobreza extrema). Los traumas provocados por la persona incluyen dos tipos, los traumas de un solo episodio o tipo I (p.ej. violación) y los traumas complejos. A su vez, los complejos incluyen dos tipos: el tipo II, episodios repetidos y similares que cesan (p. ej. abuso sexual) y el tipo III, episodios repetidos y similares que continúan (p.ej. racismo). En esta clasificación, el trauma acumulativo sería el tipo IV, que sería la resultante de los traumas anteriores, la combinación de todos ellos (véase figura 1).

\section{Correlato fisiológico/psicológico}

En lo referente a los efectos del estrés sobre el organismo, tanto a nivel fisiológico como psicológico, es ampliamente reconocido los efectos nocivos del estrés postraumático (Chu, Lieberman, 2010; Galloucis, Silverman y Francek, 2000; Haslam y Mallon, 2003; Hegadoren, Lasiuk y Coupland, 2006; Heim y Nemeroff, 2009) y, en los últimos años, también se ha investigado sobre los efectos del estrés acumulativo.

De manera más detallada, el cerebro es el órgano clave de la respuesta al estrés, ya que determina lo que es amenazador y, por tanto, potencialmente estresante, así como las respuestas fisiológicas y comportamentales, que pueden ser adaptativas 


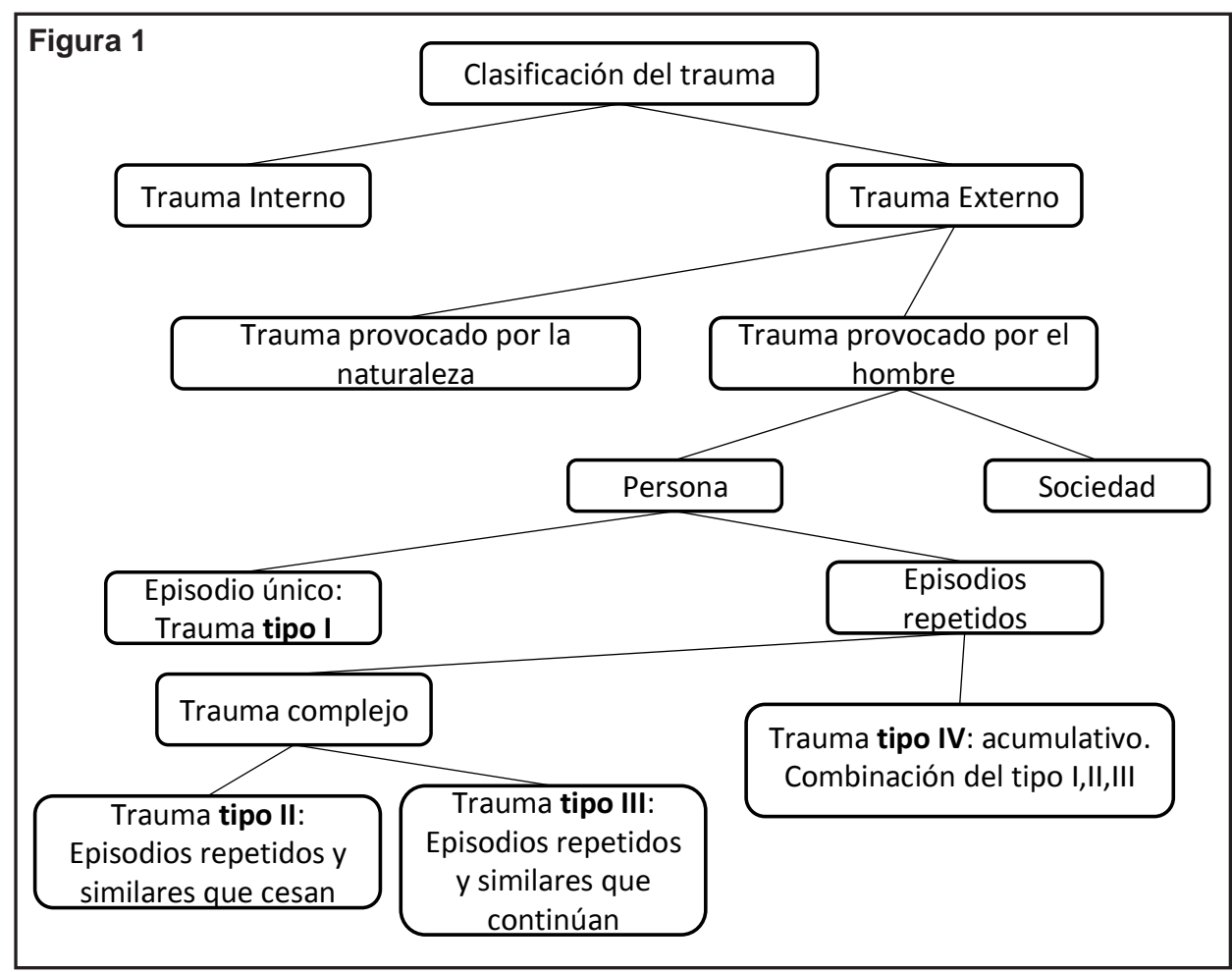

o desadaptativas (Calabrese et al., 2007). El estrés afecta las vías de comunicación que unen el cerebro con, por un lado, los sistemas cardiovascular e inmunológico y, por otro lado, con los mecanismos neurales y endocrinos (Mc Ewen, 2007). Más allá de la respuesta de huir o luchar (flight-or-fight) al estrés del incidente crítico, hay acontecimientos de la vida diaria que producen un tipo de estrés acumulativo $\mathrm{y}$, con el paso del tiempo, provocan un desgaste en el cuerpo, la llamada carga alostática (Marloes et al., 2009).

En cambio, a corto plazo, las hormonas asociadas con el estrés protegen al cuerpo y promueven la adaptación o alostasis (Mc Ewen, 2007). El cerebro es un objetivo-diana del estrés, y el hipocampo es la primera región del cerebro, además del hipotálamo, que ha sido reconocida como un objetivo-diana de los glucocorticoides. Las hormonas del estrés y el estrés en sí producen efectos tanto adaptativos como desadaptativos en esta región del cerebro de manera continua. Incluso los primeros sucesos vitales ya comienzan a influir en los patrones de la emotividad y en la respuesta al estrés que tendrá la persona a lo largo de su vida. El hipocampo, la amígdala y la corteza prefrontal sufren una remodelación estructural inducida por el estrés, que altera las respuestas conductuales y fisiológicas (Marloes et al. 2009)

Lo mismo sucede con el recuerdo de las experiencias traumáticas. El aumento 
de la memoria declarativa es adaptativo, por el evidente beneficio para la supervivencia, pero puede ser desadaptativo, culminando en estrés postraumático. Las hormonas del estrés mejoran la consolidación de los recuerdos adversos, pero también se cree que tienen efectos inmediatos en los procesos atencionales, sensoriales y mnemotécnicos de formación de la memoria (Admon et al., 2009). El estrés asociado al incidente crítico afecta a los correlatos neuronales de la formación de la memoria de un modo inesperado (Chen et al., 2010). Deteriora el aprendizaje y la memoria a través de mecanismos que alteran la integridad de las espinas dendríticas del hipocampo. Estos descubrimientos pueden explicar los mecanismos neurológicos subyacentes en la etiología del trauma psicológico.

\section{La Escala de Trauma Acumulativo}

Aunque, existen varios instrumentos que evalúan la presencia y la intensidad del trauma, el inconveniente surge cuando comparten las mismas limitaciones que la literatura científica sobre el estrés y el trauma, es decir, la mayoría de los instrumentos psicométricos no se basan en una taxonomía comprensiva integral, no ofrecen una definición clara del estrés traumático y de sus tipos (Kira et al., 2008) o simplemente se circunscriben a la definición DSM-IV-TR. Por otro lado, la única escala que mide el trauma acumulativo es la de Kira et al (2008) en inglés. Se trata de una escala autoadministrada (véase anexo) compuesta por 33 ítems que evalúan diferentes tipos de experiencias traumáticas. El sujeto debe puntuar dos aspectos de cada ítem, la frecuencia de presentación (que se puntúa en una escala Likert de 5 puntos) y la intensidad vivida (que se puntúa en una escala Likert de 7 puntos)

La traducción y adaptación lingüística del instrumento al español se realizó usando un complejo procedimiento de traducción-retraducción, la traducción inversa conceptual (Sartorius y Kuyken, 1994). La versión resultante se aplicó de forma voluntaria a un grupo piloto de 22 sujetos (13 estudiantes de la escuela de policía de Catalunya y 9 estudiantes de psicología). Ello permitió que usuarios no familiarizados con el instrumento (y posibles sujetos diana en su aplicación futura) lo probaran y expresaran sus comentarios. Con ello se identificaron aspectos mejorables que no habían sido captados en la versión inicial. A nivel anecdótico, estos sujetos encontraron dos dificultades con nuestra fiel traducción que nos parecieron legítimas y nos llevaron a consultar al Dr. Ibrahim A. Kira. Resultó que se trataba de errores en la publicación del artículo en inglés, y nos mandó la versión correcta, su manuscrito. Por supuesto, la versión en castellano corrige estos errores de la publicación original en inglés.

\section{A modo de conclusión}

Aunque el estrés y el trauma cuentan ya con una extensa literatura psicológica, la noción de trauma acumulativo supone una aportación importante a nivel 
conceptual y de gran relevancia clínica. En especial, se cree que algunas profesiones (p. ej., bomberos, policía) pueden llegar a experimentar este tipo de trauma por las características de su actividad. Al disponer de un instrumento, primero en inglés y ahora en español, se potencia la posibilidad de estudiar esta modalidad del sufrimiento humano de una forma más sistemática, estimando su prevalencia en distintas profesiones de riesgo y explorando su influencia en la salud y la enfermedad.

\footnotetext{
En este artículo se revisan las definiciones al uso de estrés y trauma, incluyendo la noción de trauma acumulativo. Seguidamente, se presenta la versión española de la primera escala existente que lo mide: la Escala de Trauma Acumulativo (CTS) de Kira et al (2008). Para obtener esta versión se ha seguido un proceso de traducciónretraducción y también se ha aplicado a dos grupos reducidos de sujetos a modo de prueba piloto.

Palabras clave: Trauma, estrés
}

\section{Referencias bibliográficas}

Admon, R.; Lubin, G.; Stern, O.; Rosenberg, K.; Sela, L.; Ben-Ami, H. \& Hendler, T. (2009), Human vulnerability to stress depends on amygdala's predisposition and hippocampal plasticity. PNAS, 106 (33), 14120-14125.

American Psychiatric Association. (2002). DSM-IV-TR. Manual diagnóstico y estadístico de los trastornos mentales. Ed. Masson: Barcelona.

Bracken, P. (2002). Trauma: Culture, meaning and philosophy. London: Whurr Publishers.

Briere, J. \& Scott, C. (2006). Principles of trauma therapy. A guide to symptoms, evaluation and treatment. London: Sage Publications.

Briere, J.(1998). Psychological Assessment of Adult Posttraumatic States. Ed. American Psychological Association: Washington.

Briere, J., \& Spinazzola, J. (2005). Phenomenology and psychological assessment of complex posttraumatic states. Journal of Traumatic Stress, 18 (5), 401-412.

Calabrese, E.; Bachmann, K.; Bailer, A. et al. (2007). Biological stress response terminology: Integrating the concepts of adaptive response and preconditioning stress within a hermetic dose-response framework. Toxicology and Applied Pharmacology, 222 (1), 122-128.

Chen, Y.; Rex, C.; Rice, C.; Dubé, C.; Gall, C.; Lynch, G. \& Baram, T. (2010). Correlated memory defects and hippocampal dendritic spine loss after acute stress involve corticotropin-releasing hormone signaling. Proceedings of the National Academy of Sciences, 107 (29), 13123-13128.

Chiappeli, F.; Manfrini, E.; Franceschi, C.; Cossarizza, A. \& Black, K. (1994). Steroid regulation in cytokines: relevance for Th1 to Th2 shift? (pp. 204-215). En: de Kloet, E.; Azmitia, E. \& Landfield, P. (Eds.). Annals of the New York Academy of Sciences: Vol. 746. Brain corticosteroid receptors: Studies on the mechanisms, functions, and neurotoxicity of corticosteroid action. New York: New York Academy of Sciences.

Chu, A. \& Lieberman, A. (2010). Clinical implications of traumatic stress from birth to age five. Annual Review Clinical Psychology, 6,469-494

Dohrenwend, B. P. (2000). The role of adversity and stress in psychopathology: Some evidence and its implications for theory and research. Journal of Health \& Social Behavior, 41 (1), 1-19.

Dulmus, C. \& Hilarski, C. (2003). When Stress Constitutes Trauma and Trauma Constitutes Crisis: The StressTrauma-Crisis Continuum. Brief Treatment and Crisis Intervention, 3 (1), 27-35 
Ehlers, A.; Bisson, J.; Clark, D.; Creamer, M.; Pilling, S.; Richards, D.; Schnurr, P.; Turner, S. \& Yule, W. (2010). Do all psychological treatments really work the same in posttraumatic stress disorder? Clinical Psychology Review, 30 (2), 269-276

Everly, G. \& Mitchell, J. (1999). Critical Incident Stress Management (CISM): A new era and standard of care in crisis intervention. Ellicott City: Chevron Publishing.

Figley, C. (2002). Treating compassion fatigue. Ed. Brunner-Routledge: New York.

Flannery, R. (1999). Critical Incident Stress Management and the Assaulted Staff Action Program. International Journal of Emergency Mental Health, 1 (2), 103-108.

Folkman, S. \& Moskowitz, J. T. (2004). Coping: pitfalls and promises. Annual Review of Psychology, 55, 745-774.

Ford, J.; Stockton, P.; Kaltman, S. \& Green, B. (2006). Disorders of extreme stress (DESNOS) symptoms are associated with type and severity of interpersonal trauma exposure in a sample of healthy young women. Journal of Interpersonal Violence, 21 (11), 1399-1416.

Galloucis, M.; Silverman, M. \& Francek, H. (2000). The impact of trauma exposure on the cognitive schemas of a sample of paramedics. International Journal of Emergency Health, 2, 5-18.

Giacaman, R.; Abu-Rmeileh, N.; Husseini, A.; Saab, H. \& Boyce, W. (2007). Humiliation: the invisible trauma of war for Palestinian youth. Public Health, 121(8), 563-571.

Gorman, W. (2001). Refugee survivors of torture: Trauma and treatment. Professional Psychology: Research and Practice, 32, 443-451.

Haslam, C.\& Mallon, K. (2003). A preliminary investigation of post-traumatic stress symptoms among firefighters. Work \& Stress, 17, 277-285.

Hegadoren, K.; Lasiuk, G. \& Coupland, N. (2006). Posttraumatic stress disorder part III: health effects of interpersonal violence among women. Perspectives in Psychiatric Care, 42 (3), 163-173.

Heim, C.\& Nemeroff, C. (2009). Neurobiology of Posttraumatic stress disorder. CNS Spectrums, 14 (suppl. 1), 1324.

Jenkins, S. \& Baird, S. (2002). Secondary traumatic stress and vicarious trauma: A validation study. Journal of Traumatic Stress, 15, 423-432.

Joseph, R. (1998). Traumatic amnesia, repression, and hippocampus injury due to emotional stress, corticosteroids and enkephalins. Child Psychiatry and Human Development, 29, 169-185.

Kira, I. (2001). Taxonomy of Trauma and Trauma Assessment. Traumatology, 7 (2), 73-86.

Kira, I.; Lewandowski, L.; Templin, T.; Ramaswamy, V.; Ozkan, V. \& Mohanesh, J. (2008). Measuring Cumulative Trauma Dose, Types, and Profiles Using a Development-Based Taxonomy of Traumas. Traumatology, 14 (2), 62-87.

Larsen, D.; Stamm, B. \& Davis, K. (2002). Telehealth for prevention and intervention of the negative effects of caregiving. Traumatic Stress Points, 16 (4).

Lazarus, R. \& Folkman, S. (1984). Stress, Appraisal, and Coping. New York: Springer.

Lazarus, R. S. (1984). Puzzles in the study of daily hassles. Journal of Behavioral Medicine, 7 (4), 375-389.

Lewis, S. \& Roberts, A. (2002). Crisis assessment tools (pp. 208-212). En: Roberts, A. \& Greene, G. (Eds.). Social workers desk reference. New York: Oxford University Press.

Linden, J. (2007). Hypnosis in childhood trauma (pp. 135-160). En: Wester, W. \& Sugarman, L. (Eds). Therapeutic Hypnosis with Children and Adolescents. Vermont: Crown House Publishing.

Lykes, M. B. (2000). Possible contributions of a psychology of liberation: Whither health and human rights? Journal of Health Psychology, 5(3), 383-397.

Marloes, J.; Henckens, A.; Erno, J.; Hermans, J. Pu, Z.; Joëls, M \& Fernández, G. (2009). Stressed memories: how acute stress affects memory formation in humans. The Journal of Neuroscience, 29 (32), 10111-10119.

McCann, L. \& Pearlman, L. (1990). Psychological trauma and the adult survivor: theory, therapy, and transformation. New York: Brunner Routledge.

McEwen, B. (1998). Protective and damaging effects of stress mediators. New England Journal of Medicine,338, 171-179.

McEwen, B. (2007). Physiology and neurobiology of stress and adaptation: central role of the brain. Physiological Reviews. 87, 873-904.

Mitchell, J. (2003). Crisis Intervention and Critical Incident Stress Management: Aresearch summary. Ellicott City, MD: International Critical Incident Stress Foundation.

North, C.; Suris, A.; Davis, M. \& Smith, R. (2009). Toward Validation of the Diagnosis of Posttraumatic Stress Disorder. American Journal of Psychiatry, 166 (1), 34-41.

Ray, S. (2008). Evolution of Posttraumatic Stress Disorder and Future Directions. Archives of Psychiatric Nursing, $22(4), 217-225$. 
Resick, P. (2001). Stress and trauma. New York: Psychology Press.

Robinson, J.; Clements, K. \& Land, C. (2003). Workplace stress among psychiatric nurses: Prevalence, distribution, correlates, and predictors. Journal of Psychosocial Nursing and Mental Health Services, 41, 32-51.

Sabin-Farrell, R. \& Turpin, G. (2003). Vicarious traumatization: Implication for the mental health of health workers. Clinical Psychology Review, 23, 449-480.

Sachs-Ericsson, N.; Blazer, D.; Plant, E. \& Arnow, B. (2005). Childhood sexual abuse and the 1-year prevalence of medical problems in the National Comorbidity Survey. Health Psychology, 24, 32-40.

Sartorius, N. \& Kuyken, W. (1994). Translation of health status instruments (p.1-18). En: Orley, J. \& Kuyken, W. (Eds.). Quality of life assessment: International perspectives. Berlin: Springer.

Segerstrom, S. \& Miller, G. (2004). Psychological stress and human immune system: A meta-analytic study of 30 years of inquiry. Psychological Bulletin, 130, 601-630.

Selye, H. (1950). Stress and the general adaptation syndrome. British Medical Journal, 1 (4667), 1383-1392.

Selye, H. (1973). The evolution of the stress concept. American Scientist, 61 (6), 692-699.

Summerfield, D. (2004). Cross-cultural perspectives on the medicalisation of human suffering (pp. 1-15). En: Rosen, G. (Ed.). Posttraumatic stress disorder: Issues and controversies. New York: John Wiley.

Taylor, R. \& Roberts, D. (1995). Kinship support and maternal and adolescent well-being in economically disadvantaged African-American families. Child Development, 66 (6), 1585-1597.

Taylor, S. \& Stanton, A. (2007). Coping resources, coping processes, and mental health. Annual Review of Clinical Psychology, 3, 129-153.

van der Kolk, B.; McFarlane, A. \& Weisaeth, L. (1996). Traumatic stress. The effects of overwhelming experience on mind, body, and society. New York: Guilford Press.

Zuckerman, M. (1999). Vulnerability to Psychopathology: A biosocial model. American Psychological Association: Washington DC. 


\section{Anexo}

\section{Cumulative Trauma Scale (short form)}

-Versión en español-

Mucha gente ha experimentado diferentes tipos de eventos y situaciones a lo largo de su vida. Los siguientes ítems le preguntarán acerca de algunos eventos determinados. Por favor indique cuántas veces le han sucedido. Y sólo si le ha sucedido, conteste cómo le ha afectado.

\section{1.- He visto o he sufrido desastres naturales, por ejemplo, huracanes, tornados} 0 inundaciones.

\begin{tabular}{|c|c|c|c|c|}
\hline Nunca & Una vez & Dos veces & Tres veces & Muchas veces \\
\hline
\end{tabular}

Si esto le ha sucedido, ¿cómo le ha afectado?

\begin{tabular}{|c|c|c|c|c|c|c|}
\hline $\begin{array}{c}\text { Extremadamente } \\
\text { positivo }\end{array}$ & $\begin{array}{c}\text { Muy } \\
\text { positivo }\end{array}$ & $\begin{array}{c}\text { Algo } \\
\text { positivo }\end{array}$ & Neutro & $\begin{array}{c}\text { Algo } \\
\text { negativo }\end{array}$ & $\begin{array}{c}\text { Muy } \\
\text { negativo }\end{array}$ & $\begin{array}{c}\text { Extremadamente } \\
\text { Negativo }\end{array}$ \\
\hline 1 & 2 & 3 & 4 & 5 & 6 & 7 \\
\hline
\end{tabular}

\section{2.- He sufrido accidentes con riesgo vital; p.ej. accidentes de tráfico}

\begin{tabular}{|c|c|c|c|c|}
\hline Nunca & Una vez & Dos veces & Tres veces & Muchas veces \\
\hline
\end{tabular}

Si esto le ha sucedido, ¿cómo le ha afectado?

\begin{tabular}{|c|c|c|c|c|c|c|}
\hline $\begin{array}{c}\text { Extremadamente } \\
\text { positivo }\end{array}$ & $\begin{array}{c}\text { Muy } \\
\text { positivo }\end{array}$ & $\begin{array}{c}\text { Algo } \\
\text { positivo }\end{array}$ & Neutro & $\begin{array}{c}\text { Algo } \\
\text { negativo }\end{array}$ & $\begin{array}{c}\text { Muy } \\
\text { negativo }\end{array}$ & $\begin{array}{c}\text { Extremadamente } \\
\text { Negativo }\end{array}$ \\
\hline 1 & 2 & 3 & 4 & 5 & 6 & 7 \\
\hline
\end{tabular}

\section{3.- Me he visto involucrado o he presenciado un combate o una guerra.}

\begin{tabular}{|c|c|c|c|c|}
\hline Nunca & Una vez & Dos veces & Tres veces & Muchas veces \\
\hline
\end{tabular}

Si esto le ha sucedido, ¿cómo le ha afectado?

\begin{tabular}{|c|c|c|c|c|c|c|}
\hline $\begin{array}{c}\text { Extremadamente } \\
\text { positivo }\end{array}$ & $\begin{array}{c}\text { Muy } \\
\text { positivo }\end{array}$ & $\begin{array}{c}\text { Algo } \\
\text { positivo }\end{array}$ & Neutro & $\begin{array}{c}\text { Algo } \\
\text { negativo }\end{array}$ & $\begin{array}{c}\text { Muy } \\
\text { negativo }\end{array}$ & $\begin{array}{c}\text { Extremadamente } \\
\text { Negativo }\end{array}$ \\
\hline 1 & 2 & 3 & 4 & 5 & 6 & 7 \\
\hline
\end{tabular}

4.- He sufrido la muerte súbita de uno de mis padres, de un íntimo amigo o de un ser querido.

\begin{tabular}{|c|c|c|c|c|}
\hline Nunca & Una vez & Dos veces & Tres veces & Muchas veces \\
\hline
\end{tabular}

Si esto le ha sucedido, ¿cómo le ha afectado?

\begin{tabular}{|c|c|c|c|c|c|c|}
\hline $\begin{array}{c}\text { Extremadamente } \\
\text { positivo }\end{array}$ & $\begin{array}{c}\text { Muy } \\
\text { positivo }\end{array}$ & $\begin{array}{c}\text { Algo } \\
\text { positivo }\end{array}$ & Neutro & $\begin{array}{c}\text { Algo } \\
\text { negativo }\end{array}$ & $\begin{array}{c}\text { Muy } \\
\text { negativo }\end{array}$ & $\begin{array}{c}\text { Extremadamente } \\
\text { Negativo }\end{array}$ \\
\hline 1 & 2 & 3 & 4 & 5 & 6 & 7 \\
\hline
\end{tabular}


5.- He experimentado un acontecimiento que amenazó o incapacitó permanentemente la vida de un ser querido.

\begin{tabular}{|c|c|c|c|c|c|c|}
\hline Nunca & \multicolumn{2}{|c|}{ Una vez } & Dos veces & \multicolumn{2}{|c|}{ Tres veces } & Muchas veces \\
\hline \multicolumn{7}{|c|}{ Si esto le ha sucedido, ¿cómo le ha afectado? } \\
\hline $\begin{array}{c}\text { Extremadamente } \\
\text { positivo }\end{array}$ & $\begin{array}{c}\text { Muy } \\
\text { positivo }\end{array}$ & $\begin{array}{c}\text { Algo } \\
\text { positivo }\end{array}$ & Neutro & $\begin{array}{c}\text { Algo } \\
\text { negativo }\end{array}$ & $\begin{array}{c}\text { Muy } \\
\text { negativo }\end{array}$ & $\begin{array}{c}\text { Extremadamente } \\
\text { Negativo }\end{array}$ \\
\hline 1 & 2 & 3 & 4 & 5 & 6 & 7 \\
\hline
\end{tabular}

6.- He padecido una enfermedad potencialmente mortal o un acontecimiento que produce una incapacidad permanente.; p. ej. cáncer, accidente vascular, una grave enfermedad crónica.

\begin{tabular}{|l|c|c|c|c|} 
Nunca & Una vez & Dos veces & Tres veces & Muchas veces \\
\hline
\end{tabular}

Si esto le ha sucedido, ¿cómo le ha afectado?

\begin{tabular}{|c|c|c|c|c|c|c|}
\hline $\begin{array}{c}\text { Extremadamente } \\
\text { positivo }\end{array}$ & $\begin{array}{c}\text { Muy } \\
\text { positivo }\end{array}$ & $\begin{array}{c}\text { Algo } \\
\text { positivo }\end{array}$ & Neutro & $\begin{array}{c}\text { Algo } \\
\text { negativo }\end{array}$ & $\begin{array}{c}\text { Muy } \\
\text { negativo }\end{array}$ & $\begin{array}{c}\text { Extremadamente } \\
\text { Negativo }\end{array}$ \\
\hline 1 & 2 & 3 & 4 & 5 & 6 & 7 \\
\hline
\end{tabular}

7.- He sufrido un robo o asalto a mano armada.

\begin{tabular}{|c|c|c|c|c|}
\hline Nunca & Una vez & Dos veces & Tres veces & Muchas veces \\
\hline
\end{tabular}

Si esto le ha sucedido, ¿cómo le ha afectado?

\begin{tabular}{|c|c|c|c|c|c|c|}
\hline $\begin{array}{c}\text { Extremadamente } \\
\text { positivo }\end{array}$ & $\begin{array}{c}\text { Muy } \\
\text { positivo }\end{array}$ & $\begin{array}{c}\text { Algo } \\
\text { positivo }\end{array}$ & Neutro & $\begin{array}{c}\text { Algo } \\
\text { negativo }\end{array}$ & $\begin{array}{c}\text { Muy } \\
\text { negativo }\end{array}$ & $\begin{array}{c}\text { Extremadamente } \\
\text { Negativo }\end{array}$ \\
\hline 1 & 2 & 3 & 4 & 5 & 6 & 7 \\
\hline
\end{tabular}

8.- He sido testigo de un asalto grave hacia un conocido o un extraño (fue tiroteado, apuñalado o severamente golpeado).

\begin{tabular}{|l|c|c|c|c|} 
Nunca & Una vez & Dos veces & Tres veces & Muchas veces \\
\hline
\end{tabular}

Si esto le ha sucedido, ¿cómo le ha afectado?

\begin{tabular}{|c|c|c|c|c|c|c|}
\hline $\begin{array}{c}\text { Extremadamente } \\
\text { positivo }\end{array}$ & $\begin{array}{c}\text { Muy } \\
\text { positivo }\end{array}$ & $\begin{array}{c}\text { Algo } \\
\text { positivo }\end{array}$ & Neutro & $\begin{array}{c}\text { Algo } \\
\text { negativo }\end{array}$ & $\begin{array}{c}\text { Muy } \\
\text { negativo }\end{array}$ & $\begin{array}{c}\text { Extremadamente } \\
\text { Negativo }\end{array}$ \\
\hline 1 & 2 & 3 & 4 & 5 & 6 & 7 \\
\hline
\end{tabular}

9.- He sido amenazado de muerte o de ser gravemente herido/apalizado.

\begin{tabular}{|c|c|c|c|c|c|c|}
\hline Nunca & \multicolumn{2}{|c|}{ Una vez } & Dos veces & \multicolumn{2}{|c|}{ Tres veces } & Muchas veces \\
\hline \multicolumn{7}{|c|}{ Si esto le ha sucedido, ¿cómo le ha afectado? } \\
\hline $\begin{array}{c}\text { Extremadamente } \\
\text { positivo }\end{array}$ & $\begin{array}{c}\text { Muy } \\
\text { positivo }\end{array}$ & $\begin{array}{c}\text { Algo } \\
\text { positivo }\end{array}$ & Neutro & $\begin{array}{c}\text { Algo } \\
\text { negativo }\end{array}$ & $\begin{array}{c}\text { Muy } \\
\text { negativo }\end{array}$ & $\begin{array}{c}\text { Extremadamente } \\
\text { Negativo }\end{array}$ \\
\hline 1 & 2 & 3 & 4 & 5 & 6 & 7 \\
\hline
\end{tabular}


10.- He sufrido abusos físicos, empujado hasta resultar herido o golpeado por un cuidador, p.ej. un padre.

\begin{tabular}{|c|c|c|c|c|c|c|}
\hline Nunca & \multicolumn{2}{|c|}{ Una vez } & Dos veces & \multicolumn{2}{|c|}{ Tres veces } & Muchas veces \\
\hline \multicolumn{7}{|c|}{ Si esto le ha sucedido, ¿cómo le ha afectado? } \\
\hline $\begin{array}{l}\text { Extremadamente } \\
\text { positivo }\end{array}$ & $\begin{array}{c}\text { Muy } \\
\text { positivo }\end{array}$ & $\begin{array}{c}\text { Algo } \\
\text { positivo }\end{array}$ & Neutro & $\begin{array}{c}\text { Algo } \\
\text { negativo }\end{array}$ & $\begin{array}{c}\text { Muy } \\
\text { negativo }\end{array}$ & $\begin{array}{c}\text { Extremadamente } \\
\text { Negativo }\end{array}$ \\
\hline 1 & 2 & 3 & 4 & 5 & 6 & 7 \\
\hline
\end{tabular}

11.- He visto u oído a uno de mis padres/cuidadores golpeando, hiriendo y/o amenazando al otro padre/cuidador.

\begin{tabular}{|l|c|c|c|c|} 
Nunca & Una vez & Dos veces & Tres veces & Muchas veces \\
\hline
\end{tabular}

Si esto le ha sucedido, ¿cómo le ha afectado?

\begin{tabular}{|c|c|c|c|c|c|c|}
\hline $\begin{array}{c}\text { Extremadamente } \\
\text { positivo }\end{array}$ & $\begin{array}{c}\text { Muy } \\
\text { positivo }\end{array}$ & $\begin{array}{c}\text { Algo } \\
\text { positivo }\end{array}$ & Neutro & $\begin{array}{c}\text { Algo } \\
\text { negativo }\end{array}$ & $\begin{array}{c}\text { Muy } \\
\text { negativo }\end{array}$ & $\begin{array}{c}\text { Extremadamente } \\
\text { Negativo }\end{array}$ \\
\hline 1 & 2 & 3 & 4 & 5 & 6 & 7 \\
\hline
\end{tabular}

12.- He sido empujado a mantener relaciones sexuales por alguien mayor que yo.

\begin{tabular}{|c|c|c|c|c|}
\hline Nunca & Una vez & Dos veces & Tres veces & Muchas veces \\
\hline
\end{tabular}

Si esto le ha sucedido, ¿cómo le ha afectado?

\begin{tabular}{|c|c|c|c|c|c|c|}
\hline $\begin{array}{c}\text { Extremadamente } \\
\text { positivo }\end{array}$ & $\begin{array}{c}\text { Muy } \\
\text { positivo }\end{array}$ & $\begin{array}{c}\text { Algo } \\
\text { positivo }\end{array}$ & Neutro & $\begin{array}{c}\text { Algo } \\
\text { negativo }\end{array}$ & $\begin{array}{c}\text { Muy } \\
\text { negativo }\end{array}$ & $\begin{array}{c}\text { Extremadamente } \\
\text { Negativo }\end{array}$ \\
\hline 1 & 2 & 3 & 4 & 5 & 6 & 7 \\
\hline
\end{tabular}

13.- He sufrido abusos sexuales, he sido violado, o me he visto envuelto en relaciones sexuales no deseadas con una o más personas.

\begin{tabular}{|c|c|c|c|c|}
\hline Nunca & Una vez & Dos veces & Tres veces & Muchas veces \\
\hline
\end{tabular}

Si esto le ha sucedido, ¿cómo le ha afectado?

\begin{tabular}{|c|c|c|c|c|c|c|}
\hline $\begin{array}{c}\text { Extremadamente } \\
\text { positivo }\end{array}$ & $\begin{array}{c}\text { Muy } \\
\text { positivo }\end{array}$ & $\begin{array}{c}\text { Algo } \\
\text { positivo }\end{array}$ & Neutro & $\begin{array}{c}\text { Algo } \\
\text { negativo }\end{array}$ & $\begin{array}{c}\text { Muy } \\
\text { negativo }\end{array}$ & $\begin{array}{c}\text { Extremadamente } \\
\text { Negativo }\end{array}$ \\
\hline 1 & 2 & 3 & 4 & 5 & 6 & 7 \\
\hline
\end{tabular}

\section{4.- He sido encarcelado y/o torturado.}

\begin{tabular}{|c|c|c|c|c|}
\hline Nunca & Una vez & Dos veces & Tres veces & Muchas veces \\
\hline
\end{tabular}

Si esto le ha sucedido, ¿cómo le ha afectado?

\begin{tabular}{|c|c|c|c|c|c|c|}
\hline $\begin{array}{c}\text { Extremadamente } \\
\text { positivo }\end{array}$ & $\begin{array}{c}\text { Muy } \\
\text { positivo }\end{array}$ & $\begin{array}{c}\text { Algo } \\
\text { positivo }\end{array}$ & Neutro & $\begin{array}{c}\text { Algo } \\
\text { negativo }\end{array}$ & $\begin{array}{c}\text { Muy } \\
\text { negativo }\end{array}$ & $\begin{array}{c}\text { Extremadamente } \\
\text { Negativo }\end{array}$ \\
\hline 1 & 2 & 3 & 4 & 5 & 6 & 7 \\
\hline
\end{tabular}


15.- Mi madre me abandonó o se separó de mi cuando yo era joven.

\begin{tabular}{|c|c|c|c|c|c|c|}
\hline Nunca & \multicolumn{2}{|c|}{ Una vez } & \multicolumn{2}{c|}{ Dos veces } & Tres veces & Muchas veces \\
\hline $\begin{array}{c}\text { esto le ha sucedido, ¿cómo le ha afectado? } \\
\text { positivo }\end{array}$ & $\begin{array}{c}\text { Muy } \\
\text { positivo }\end{array}$ & $\begin{array}{c}\text { Algo } \\
\text { positivo }\end{array}$ & Neutro & $\begin{array}{c}\text { Algo } \\
\text { negativo }\end{array}$ & $\begin{array}{c}\text { Muy } \\
\text { negativo }\end{array}$ & $\begin{array}{c}\text { Extremadamente } \\
\text { Negativo }\end{array}$ \\
\hline 1 & 2 & 3 & 4 & 5 & 6 & 7 \\
\hline
\end{tabular}

16.- Mi padre me abandonó o se separó de mi cuando yo era joven.

\begin{tabular}{|c|c|c|c|c|c|c|}
\hline Nunca & \multicolumn{2}{|c|}{ Una vez } & Dos veces & \multicolumn{2}{|c|}{ Tres veces } & Muchas veces \\
\hline \multicolumn{7}{|c|}{ Si esto le ha sucedido, ¿cómo le ha afectado? } \\
\hline $\begin{array}{c}\text { Extremadamente } \\
\text { positivo }\end{array}$ & $\begin{array}{c}\text { Muy } \\
\text { positivo }\end{array}$ & $\begin{array}{c}\text { Algo } \\
\text { positivo }\end{array}$ & Neutro & $\begin{array}{c}\text { Algo } \\
\text { negativo }\end{array}$ & $\begin{array}{c}\text { Muy } \\
\text { negativo }\end{array}$ & $\begin{array}{c}\text { Extremadamente } \\
\text { Negativo }\end{array}$ \\
\hline 1 & 2 & 3 & 4 & 5 & 6 & 7 \\
\hline
\end{tabular}

17.- He sido menospreciado, amenazado, o discriminado por las actitudes negativas, estereotipos o acciones de otros, a causa de mi etnia, raza, cultura, religión o nacionalidad de origen.

\begin{tabular}{|c|c|c|c|c|}
\hline Nunca & Una vez & Dos veces & Tres veces & Muchas veces \\
\hline
\end{tabular}

Si esto le ha sucedido, ¿cómo le ha afectado?

\begin{tabular}{|c|c|c|c|c|c|c|}
\hline $\begin{array}{c}\text { Extremadamente } \\
\text { positivo }\end{array}$ & $\begin{array}{c}\text { Muy } \\
\text { positivo }\end{array}$ & $\begin{array}{c}\text { Algo } \\
\text { positivo }\end{array}$ & Neutro & $\begin{array}{c}\text { Algo } \\
\text { negativo }\end{array}$ & $\begin{array}{c}\text { Muy } \\
\text { negativo }\end{array}$ & $\begin{array}{c}\text { Extremadamente } \\
\text { Negativo }\end{array}$ \\
\hline 1 & 2 & 3 & 4 & 5 & 6 & 7 \\
\hline
\end{tabular}

18.- Mis padres se divorciaron/separaron.

\begin{tabular}{|c|c|c|c|c|}
\hline Nunca & Una vez & Dos veces & Tres veces & Muchas veces \\
\hline
\end{tabular}

Si esto le ha sucedido, ¿cómo le ha afectado?

\begin{tabular}{|c|c|c|c|c|c|c|}
\hline $\begin{array}{c}\text { Extremadamente } \\
\text { positivo }\end{array}$ & $\begin{array}{c}\text { Muy } \\
\text { positivo }\end{array}$ & $\begin{array}{c}\text { Algo } \\
\text { positivo }\end{array}$ & Neutro & $\begin{array}{c}\text { Algo } \\
\text { negativo }\end{array}$ & $\begin{array}{c}\text { Muy } \\
\text { negativo }\end{array}$ & $\begin{array}{c}\text { Extremadamente } \\
\text { Negativo }\end{array}$ \\
\hline 1 & 2 & 3 & 4 & 5 & 6 & 7 \\
\hline
\end{tabular}

19.- Mi raza tiene una historia de opresión, discriminación y amenaza de genocidio.

\begin{tabular}{|c|c|c|c|c|}
\hline Nunca & $\begin{array}{c}\text { Poco de } \\
\text { acuerdo }\end{array}$ & $\begin{array}{c}\text { Parcialmente } \\
\text { de acuerdo }\end{array}$ & $\begin{array}{c}\text { Bastante } \\
\text { de acuerdo }\end{array}$ & $\begin{array}{c}\text { Muy de } \\
\text { acuerdo }\end{array}$ \\
\hline
\end{tabular}

Si esto le ha sucedido, ¿cómo le ha afectado?

\begin{tabular}{|c|c|c|c|c|c|c|}
\hline $\begin{array}{c}\text { Extremadamente } \\
\text { positivo }\end{array}$ & $\begin{array}{c}\text { Muy } \\
\text { positivo }\end{array}$ & $\begin{array}{c}\text { Algo } \\
\text { positivo }\end{array}$ & Neutro & $\begin{array}{c}\text { Algo } \\
\text { negativo }\end{array}$ & $\begin{array}{c}\text { Muy } \\
\text { negativo }\end{array}$ & $\begin{array}{c}\text { Extremadamente } \\
\text { Negativo }\end{array}$ \\
\hline 1 & 2 & 3 & 4 & 5 & 6 & 7 \\
\hline
\end{tabular}


20.- He experimentado un ataque de nervios o me he sentido a punto de tenerlo (perder el control), debido a factores crónicos de estrés, aparentemente pequeños, pero recurrentes o constantes.

\begin{tabular}{|c|c|c|c|c|c|c|}
\hline Nunca & \multicolumn{2}{|c|}{ Una vez } & Dos veces & \multicolumn{2}{|c|}{ Tres veces } & Muchas veces \\
\hline \multicolumn{7}{|c|}{ Si esto le ha sucedido, ¿cómo le ha afectado? } \\
\hline $\begin{array}{c}\text { Extremadamente } \\
\text { positivo }\end{array}$ & $\begin{array}{c}\text { Muy } \\
\text { positivo }\end{array}$ & $\begin{array}{c}\text { Algo } \\
\text { positivo }\end{array}$ & Neutro & $\begin{array}{c}\text { Algo } \\
\text { negativo }\end{array}$ & $\begin{array}{c}\text { Muy } \\
\text { negativo }\end{array}$ & $\begin{array}{c}\text { Extremadamente } \\
\text { Negativo }\end{array}$ \\
\hline 1 & 2 & 3 & 4 & 5 & 6 & 7 \\
\hline
\end{tabular}

21.- Al menos uno de mis padres o hermanos-as ha participado en una guerra, combate 0 , ha sido torturado.

\begin{tabular}{|c|c|c|c|c|}
\hline Nunca & Una vez & Dos veces & Tres veces & Muchas veces \\
\hline
\end{tabular}

Si esto le ha sucedido, ¿cómo le ha afectado?

\begin{tabular}{|c|c|c|c|c|c|c|}
\hline $\begin{array}{c}\text { Extremadamente } \\
\text { positivo }\end{array}$ & $\begin{array}{c}\text { Muy } \\
\text { positivo }\end{array}$ & $\begin{array}{c}\text { Algo } \\
\text { positivo }\end{array}$ & Neutro & $\begin{array}{c}\text { Algo } \\
\text { negativo }\end{array}$ & $\begin{array}{c}\text { Muy } \\
\text { negativo }\end{array}$ & $\begin{array}{c}\text { Extremadamente } \\
\text { Negativo }\end{array}$ \\
\hline 1 & 2 & 3 & 4 & 5 & 6 & 7 \\
\hline
\end{tabular}

22.- He sufrido fracaso escolar.

\begin{tabular}{|c|c|c|c|c|}
\hline Nunca & Una vez & Dos veces & Tres veces & Muchas veces \\
\hline
\end{tabular}

Si esto le ha sucedido, ¿cómo le ha afectado?

\begin{tabular}{|c|c|c|c|c|c|c|}
\hline $\begin{array}{c}\text { Extremadamente } \\
\text { positivo }\end{array}$ & $\begin{array}{c}\text { Muy } \\
\text { positivo }\end{array}$ & $\begin{array}{c}\text { Algo } \\
\text { positivo }\end{array}$ & Neutro & $\begin{array}{c}\text { Algo } \\
\text { negativo }\end{array}$ & $\begin{array}{c}\text { Muy } \\
\text { negativo }\end{array}$ & $\begin{array}{c}\text { Extremadamente } \\
\text { Negativo }\end{array}$ \\
\hline 1 & 2 & 3 & 4 & 5 & 6 & 7 \\
\hline
\end{tabular}

23.- He sido desarraigado y obligado a desplazarme de mi entorno favorito de la ciudad, del pueblo o del país.

\begin{tabular}{|c|c|c|c|c|}
\hline Nunca & Una vez & Dos veces & Tres veces & Muchas veces \\
\hline
\end{tabular}

Si esto le ha sucedido, ¿cómo le ha afectado?

\begin{tabular}{|c|c|c|c|c|c|c|}
\hline $\begin{array}{c}\text { Extremadamente } \\
\text { positivo }\end{array}$ & $\begin{array}{c}\text { Muy } \\
\text { positivo }\end{array}$ & $\begin{array}{c}\text { Algo } \\
\text { positivo }\end{array}$ & Neutro & $\begin{array}{c}\text { Algo } \\
\text { negativo }\end{array}$ & $\begin{array}{c}\text { Muy } \\
\text { negativo }\end{array}$ & $\begin{array}{c}\text { Extremadamente } \\
\text { Negativo }\end{array}$ \\
\hline 1 & 2 & 3 & 4 & 5 & 6 & 7 \\
\hline
\end{tabular}

24.- He sido atacado, golpeado por alguien (persona más fuerte o grupo de personas), resultando herido.

\begin{tabular}{|c|c|c|c|c|}
\hline Nunca & Una vez & Dos veces & Tres veces & Muchas veces \\
\hline
\end{tabular}

Si esto le ha sucedido, ¿cómo le ha afectado?

\begin{tabular}{|c|c|c|c|c|c|c|}
\hline $\begin{array}{c}\text { Extremadamente } \\
\text { positivo }\end{array}$ & $\begin{array}{c}\text { Muy } \\
\text { positivo }\end{array}$ & $\begin{array}{c}\text { Algo } \\
\text { positivo }\end{array}$ & Neutro & $\begin{array}{c}\text { Algo } \\
\text { negativo }\end{array}$ & $\begin{array}{c}\text { Muy } \\
\text { negativo }\end{array}$ & $\begin{array}{c}\text { Extremadamente } \\
\text { Negativo }\end{array}$ \\
\hline 1 & 2 & 3 & 4 & 5 & 6 & 7 \\
\hline
\end{tabular}


25.- He sido empujado a mantener relaciones sexuales por uno de mis cuidadores/padres.

\begin{tabular}{|c|c|c|c|c|c|c|}
\hline Nunca & \multicolumn{2}{|c|}{ Una vez } & Dos veces & \multicolumn{2}{|c|}{ Tres veces } & Muchas veces \\
\hline $\begin{array}{c}\text { Extremadamente } \\
\text { positivo }\end{array}$ & $\begin{array}{c}\text { Muy } \\
\text { positivo }\end{array}$ & $\begin{array}{c}\text { Algo } \\
\text { positivo }\end{array}$ & Neutro & $\begin{array}{c}\text { Algo } \\
\text { negativo }\end{array}$ & $\begin{array}{c}\text { Muy } \\
\text { negativo }\end{array}$ & $\begin{array}{c}\text { Extremadamente } \\
\text { Negativo }\end{array}$ \\
\hline 1 & 2 & 3 & 4 & 5 & 6 & 7 \\
\hline
\end{tabular}

26.- He sido menospreciado, denigrado en mis derechos o discriminado socialmente (no por miembros de la familia), por causa de ciertas actitudes negativas, estereotipos o acciones de otros, o por instituciones, a causa de mi género.

\begin{tabular}{|l|l|l|l|l|} 
Nunca & Una vez & Dos veces & Tres veces & Muchas veces \\
\hline
\end{tabular}

Si esto le ha sucedido, ¿cómo le ha afectado?

\begin{tabular}{|c|c|c|c|c|c|c|}
\hline $\begin{array}{c}\text { Extremadamente } \\
\text { positivo }\end{array}$ & $\begin{array}{c}\text { Muy } \\
\text { positivo }\end{array}$ & $\begin{array}{c}\text { Algo } \\
\text { positivo }\end{array}$ & Neutro & $\begin{array}{c}\text { Algo } \\
\text { negativo }\end{array}$ & $\begin{array}{c}\text { Muy } \\
\text { negativo }\end{array}$ & $\begin{array}{c}\text { Extremadamente } \\
\text { Negativo }\end{array}$ \\
\hline 1 & 2 & 3 & 4 & 5 & 6 & 7 \\
\hline
\end{tabular}

27.- He sufrido un rechazo evidente o fracaso en mis relaciones.

\begin{tabular}{|c|c|c|c|c|c|c|}
\hline Nunca & \multicolumn{2}{|c|}{ Una vez } & Dos veces & \multicolumn{2}{|c|}{ Tres veces } & Muchas veces \\
\hline \multicolumn{7}{|c|}{ Si esto le ha sucedido, ¿cómo le ha afectado? } \\
\hline $\begin{array}{c}\text { Extremadamente } \\
\text { positivo }\end{array}$ & $\begin{array}{c}\text { Muy } \\
\text { positivo }\end{array}$ & $\begin{array}{c}\text { Algo } \\
\text { positivo }\end{array}$ & Neutro & $\begin{array}{c}\text { Algo } \\
\text { negativo }\end{array}$ & $\begin{array}{c}\text { Muy } \\
\text { negativo }\end{array}$ & $\begin{array}{c}\text { Extremadamente } \\
\text { Negativo }\end{array}$ \\
\hline 1 & 2 & 3 & 4 & 5 & 6 & 7 \\
\hline
\end{tabular}

28.- He sufrido la pérdida de un hijo o esposo-a.

\begin{tabular}{|c|c|c|c|c|}
\hline Nunca & Una vez & Dos veces & Tres veces & Muchas veces \\
\hline
\end{tabular}

Si esto le ha sucedido, ¿cómo le ha afectado?

\begin{tabular}{|c|c|c|c|c|c|c|}
\hline $\begin{array}{c}\text { Extremadamente } \\
\text { positivo }\end{array}$ & $\begin{array}{c}\text { Muy } \\
\text { positivo }\end{array}$ & $\begin{array}{c}\text { Algo } \\
\text { positivo }\end{array}$ & Neutro & $\begin{array}{c}\text { Algo } \\
\text { negativo }\end{array}$ & $\begin{array}{c}\text { Muy } \\
\text { negativo }\end{array}$ & $\begin{array}{c}\text { Extremadamente } \\
\text { Negativo }\end{array}$ \\
\hline 1 & 2 & 3 & 4 & 5 & 6 & 7 \\
\hline
\end{tabular}

29.- He vivido la finalización de un contrato laboral, he sido despedido o he fracasado en los negocios.

\begin{tabular}{|c|c|c|c|c|c|c|}
\hline Nunca & \multicolumn{2}{|c|}{ Una vez } & Dos veces & \multicolumn{2}{|c|}{ Tres veces } & Muchas veces \\
\hline \multicolumn{7}{|c|}{ Si esto le ha sucedido, ¿cómo le ha afectado? } \\
\hline $\begin{array}{c}\text { Extremadamente } \\
\text { positivo }\end{array}$ & $\begin{array}{c}\text { Muy } \\
\text { positivo }\end{array}$ & $\begin{array}{c}\text { Algo } \\
\text { positivo }\end{array}$ & Neutro & $\begin{array}{c}\text { Algo } \\
\text { negativo }\end{array}$ & $\begin{array}{c}\text { Muy } \\
\text { negativo }\end{array}$ & $\begin{array}{c}\text { Extremadamente } \\
\text { Negativo }\end{array}$ \\
\hline 1 & 2 & 3 & 4 & 5 & 6 & 7 \\
\hline
\end{tabular}


30.- Me he vuelto a casar.

\begin{tabular}{|c|c|c|c|c|c|c|}
\hline Nunca & \multicolumn{2}{|c|}{ Una vez } & Dos veces & \multicolumn{2}{|c|}{ Tres veces } & Muchas veces \\
\hline \multicolumn{7}{|c|}{ Si esto le ha sucedido, ¿cómo le ha afectado? } \\
\hline $\begin{array}{c}\text { Extremadamente } \\
\text { positivo }\end{array}$ & $\begin{array}{c}\text { Muy } \\
\text { positivo }\end{array}$ & $\begin{array}{c}\text { Algo } \\
\text { positivo }\end{array}$ & Neutro & $\begin{array}{c}\text { Algo } \\
\text { negativo }\end{array}$ & $\begin{array}{c}\text { Muy } \\
\text { negativo }\end{array}$ & $\begin{array}{c}\text { Extremadamente } \\
\text { Negativo }\end{array}$ \\
\hline 1 & 2 & 3 & 4 & 5 & 6 & 7 \\
\hline
\end{tabular}

31.- He crecido en el seno de una familia pobre con muchas penurias.

\begin{tabular}{|c|c|c|c|c|}
\hline Nunca & Algo pobre & $\begin{array}{c}\text { Realmente } \\
\text { pobre }\end{array}$ & Muy pobre & $\begin{array}{c}\text { Extremadamente } \\
\text { pobre }\end{array}$ \\
\hline
\end{tabular}

Si esto le ha sucedido, ¿cómo le ha afectado?

\begin{tabular}{|c|c|c|c|c|c|c|}
\hline $\begin{array}{c}\text { Extremadamente } \\
\text { positivo }\end{array}$ & $\begin{array}{c}\text { Muy } \\
\text { positivo }\end{array}$ & $\begin{array}{c}\text { Algo } \\
\text { positivo }\end{array}$ & Neutro & $\begin{array}{c}\text { Algo } \\
\text { negativo }\end{array}$ & $\begin{array}{c}\text { Muy } \\
\text { negativo }\end{array}$ & $\begin{array}{c}\text { Extremadamente } \\
\text { Negativo }\end{array}$ \\
\hline 1 & 2 & 3 & 4 & 5 & 6 & 7 \\
\hline
\end{tabular}

32.- He sido menospreciado, denigrado en mis derechos o discriminado por miembros de la familia, por ciertas actitudes negativas, estereotipos, 0 acciones, a causa de mi género.

\begin{tabular}{|c|c|c|c|c|}
\hline Nunca & Una vez & Dos veces & Tres veces & Muchas veces \\
\hline
\end{tabular}

Si esto le ha sucedido, ¿cómo le ha afectado?

\begin{tabular}{|c|c|c|c|c|c|c|}
\hline $\begin{array}{c}\text { Extremadamente } \\
\text { positivo }\end{array}$ & $\begin{array}{c}\text { Muy } \\
\text { positivo }\end{array}$ & $\begin{array}{c}\text { Algo } \\
\text { positivo }\end{array}$ & Neutro & $\begin{array}{c}\text { Algo } \\
\text { negativo }\end{array}$ & $\begin{array}{c}\text { Muy } \\
\text { negativo }\end{array}$ & $\begin{array}{c}\text { Extremadamente } \\
\text { Negativo }\end{array}$ \\
\hline 1 & 2 & 3 & 4 & 5 & 6 & 7 \\
\hline
\end{tabular}

33.- He sido discriminado a causa de mi género por personas ajenas a mi familia, las instituciones o mi comunidad.

\begin{tabular}{|c|c|c|c|c|}
\hline Nunca & Una vez & Dos veces & Tres veces & Muchas veces \\
\hline
\end{tabular}

Si esto le ha sucedido, ¿cómo le ha afectado?

\begin{tabular}{|c|c|c|c|c|c|c|}
\hline $\begin{array}{c}\text { Extremadamente } \\
\text { positivo }\end{array}$ & $\begin{array}{c}\text { Muy } \\
\text { positivo }\end{array}$ & $\begin{array}{c}\text { Algo } \\
\text { positivo }\end{array}$ & Neutro & $\begin{array}{c}\text { Algo } \\
\text { negativo }\end{array}$ & $\begin{array}{c}\text { Muy } \\
\text { negativo }\end{array}$ & $\begin{array}{c}\text { Extremadamente } \\
\text { Negativo }\end{array}$ \\
\hline 1 & 2 & 3 & 4 & 5 & 6 & 7 \\
\hline
\end{tabular}

